\title{
On the Effects of Rotation on the Passive Scalar and Kinematic Fields of Homogeneous Sheared Turbulence
}

\author{
B. Chebbi and M. Bouzaiane ${ }^{\dagger}$ \\ Laboratoire de mécanique des fluides, Département de Physique, Faculté des Sciences de Tunis \\ †Corresponding Author Email: mounir.bouzaiane@fsb.rnu.tn, mbouzaiane@yahoo.fr
}

(Received April 10, 2010; accepted May 29, 2010)

\begin{abstract}
In this work, the effect of rotation on the evolution of kinematic and passive scalar fields in two dimensional homogeneous sheared turbulence is studied using two different approaches. The first one is analytical and it consists on the resolution of differential linear equations governing the turbulence at high shear when the non linear effects are neglected. The second one is numerical and it consists on the modeling of governing equations using the most known second order models of turbulence and their numerical integration using the fourth order Runge-kutta method. In this second approach, the classic Launder Reece Rodi model, the Speziale Sarkar Gatski and the Shih Lumley models are retained for the pressure-strain correlation, pressure-scalar gradient correlation and for the time evolution equations of the kinematic and scalar dissipations. The evolution of turbulence is studied according to the dimensionless rotation number $\mathrm{R}$ which is varied from -0.75 to 0.5 . The obtained results are compared to the recent results of the DNS of Brethouwer. Both methods have confirmed the existence of asymptotic equilibrium states for dimensionless kinematic and scalar parameters.
\end{abstract}

Keywords: Turbulence with the rotation, Passive scalar field, Second order modeling, Asymptotic behavior.

\section{INTROODUCTION}

During an important period, numerous theoretical works and laboratory experiments have been performed in order to understand and predict turbulent flow behavior. In the absence of exact predictive theory of turbulence, second order modeling remains one of the more important approaches used to describe and analyze turbulent flows. Recently, second order models have served to study complex configurations of turbulent flows Khaleghi et al. (2010). It is by second order closure models also that we have studied an homogeneous sheared turbulence submitted to a stably stratification (Bouzaiane et al. 2004; 2003)

The last few years, some authors were interested to the study of the effect of rotation on turbulence. In fact, rotation is present in a larger domain of application in nature especially oceanic current and atmospheric boundary layers as well as in industrial mechanical like turbo machines.

Modeling rotating turbulent flow received considerable attention, kinematic field of homogeneous sheared turbulence has been widely studied by authors Porosova (2002), Speziale et al. (1988) and (1989). The last paper is considered by many authors as one of the most interesting work during the last two decades dedicated to the study of rotation effects on the kinematic field. On the contrary, the effects of rotation on the passive scalar field are rare. In the absence of rotation effects, experimental results of Tavoularis and Corrsin (1981) constitute one of the principal results dedicated to the study of passive scalar field in homogeneous sheared turbulence. The lack of result for transport of passive scalar field forms the motivation of this work in which peculiar attention will be accorded to the asymptotic behavior at long time evolution of dimensionless scalars parameters in addition to kinematic ones. The three sophisticated second order closure models of Launder Reece Rodi (Launder et al. 1975), Speziale Sarkar Gatski (Speziale et al. 1990) and Shih Lumley (Shih et al. 1985-b) are retained here for the pressurestrain correlation, the pressure-scalar gradient correlations and the transport equations of the kinematic and scalar dissipations of the turbulent kinetic energy and of the variance of scalar. The results of direct numerical simulation of Brethouwer (2005), which are, to our knowledge, the most interesting and recent works of the considered flow have been retained. The experimental results of Tavoularis and Corrsin (1981) are also retained in the present work. This work devoted essentially to the prediction of asymptotic equilibrium states through theoretical and numerical approaches is presented as follows: in section 2 equations of motion used in this study are introduced and the transports of second order moments are derived. In section 3, analytical solutions in the case of high shear when non linear effects are neglected are obtained. Solutions are investigated to study the 
asymptotic behavior at long time evolution of non dimensional parameters. The second order modeling of transport equations of second moments makes the object of section 4 whereas their castling in non dimensional forms and numerical integration makes the object of section 5. A peculiar attention will be accorded to in this section also to the prediction of the asymptotic equilibrium states at long time evolution. Principal obtained results in this work, are summarized in section 6 .

\section{Mathematical Consideration}

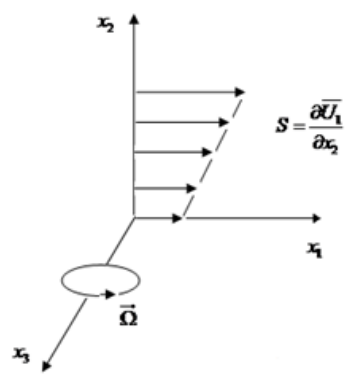

Fig. 1. Sketch of the mean velocity profile, coordinate system and the direction of rotation.

In an orthonormal cartesian coordinate system of components $\left(\mathrm{x}_{1}, \mathrm{x}_{2}, \mathrm{x}_{3}\right)$, the flow to be considered in the present work is a two dimensional (2-D) homogeneous turbulent shear flow of a viscous incompressible fluid in a rotating frame. The mean velocity $\overline{\bar{U}}=\left(\overline{U_{1}}, 0,0\right)$ is unidirectional according $\mathrm{x}_{1}$ and has a constant vertical shear rate $S=\frac{\partial \overline{U_{1}}}{\partial x_{2}}$, according $\mathrm{x}_{2}, \vec{\Omega}$ is the rotating vector and $x_{3}$ is the direction of rotation (Fig. 1). A passive scalar field with a constant mean scalar gradient $G=\frac{d \bar{T}}{d x_{2}} \quad$ is superimposed. The gradient $\mathrm{G}$ is small enough to have negligible effect on the velocity field of the turbulence. Hence only non buoyant flows are considered.

\subsection{Fundamental Equation}

The study of an incompressible turbulent shear flow is based on the continuity equation, the Navier Stokes equation and a transport equation for the passive scalar. Classically in turbulence, Reynolds decomposition (Cadiou, 1996) is introduced. The dependent variables velocity $U_{i}$, scalar $T$ and pressure $P$ are decomposed into mean parts, denoted by over bar, and fluctuating parts noted $u_{i}, p$ and $\theta$ respectively:

$U_{i}=\overline{U_{i}}+u_{i}, P=\bar{P}+p, T=\bar{T}+\theta$

The evolution equations for the fluctuating parts are obtained by introducing the above decomposition of the dependent variables and then differencing the resulting equation with the evolution equations for the mean parts. For the considered rotating homogeneous turbulent shear flow, the evolution equations of the fluctuating parts take the following forms:

$$
\begin{aligned}
& \frac{\partial u_{i}}{\partial x_{i}}=0 \\
& \frac{\partial u_{i}}{\partial t}+\overline{U_{k}} \frac{\partial u_{i}}{\partial x_{k}}=-\frac{\partial p}{\partial x_{i}}-u_{k} \frac{\partial \overline{U_{i}}}{\partial x_{k}}-2 \varepsilon_{i m p} \Omega_{m} u_{p} \\
& +\frac{\partial}{\partial x_{k}}\left(v \frac{\partial u_{i}}{\partial x_{k}}-u_{i} u_{k}+\overline{u_{i} u_{k}}\right) \\
& \frac{\partial \theta}{\partial t}+\overline{U_{k}} \frac{\partial \theta}{\partial x_{k}}=-u_{k} \frac{\partial \bar{T}}{\partial x_{k}} \\
& +\frac{\partial}{\partial x_{k}}\left(\alpha \frac{\partial \theta}{\partial x_{k}}-\theta u_{k}+\overline{\theta u_{k}}\right)
\end{aligned}
$$

Here, $\varepsilon_{i j k}$ are components of permutation tensor known also as Ricci tensor, $v$ is the kinematic viscosity and $\alpha$ is the scalar diffusivity. At this step, transport equations of second order moments are derived.

\subsection{Transport Equation}

In this section transport equations for the components $\overline{u_{i} u_{j}}$ of the Reynolds stress, the components $\overline{\theta u_{i}}$ of the turbulent scalar flux, the turbulent kinetic energy $\mathrm{K}$ and the variance of scalar $\overline{\theta^{2}}$ are written. The transport equation of the components $\overline{u_{i} u_{j}}$ of the Reynolds stress may be obtained by standard method. It can be derived from combination of the $\mathrm{i}^{\text {th }}$ and the $\mathrm{j}^{\text {th }}$ component of the momentum equation (Brethouwer 2005):

$\frac{d}{d t} \overline{u_{i} u_{j}}=P_{i j}+\phi_{i j}-\varepsilon_{i j}$

Here $\frac{d}{d t}=\frac{\partial}{\partial t}+\bar{U}_{k} \frac{\partial}{\partial x_{k}}$ is the total time derivative.

In an analogous manner an equation for the components $\overline{\theta u_{i}}$ of the turbulent scalar flux is derived:

$\frac{d}{d t} \overline{\theta u_{i}}=P_{i \theta}+\phi_{i \theta}-\varepsilon_{i \theta}$

While considering the trace of Eq. (4), we get the time evolution equation of turbulent kinetic energy $K=\overline{u_{i} u_{i}} / 2$

$\frac{d K}{d t}=P-\varepsilon$

The equation of scalar variance is associated to Eqs. (4), (5) and (6). It is derived from the transport equation of scalar fluctuation (3) and it is written in the following form:

$\frac{d \overline{\theta^{2}}}{d t}=P_{\theta}-\varepsilon_{\theta}$

In all these transport equations, terms denoted by $\mathrm{P}$ are terms of production due to mean kinematic and scalar gradients:

$P_{i j}=-\overline{u_{j} u_{k}} \frac{\partial \overline{U_{i}}}{\partial x_{k}}-\overline{u_{i} u_{k}} \frac{\partial \overline{U_{j}}}{\partial x_{k}}$ 


$$
\begin{aligned}
& -2\left(\varepsilon_{i m k} \overline{u_{j} u_{k}}+\varepsilon_{j m k} \overline{u_{i} u_{k}}\right) \Omega_{m} \\
P_{i \theta}= & -\overline{u_{i} u_{k}} \frac{\partial \bar{T}}{\partial x_{k}} \\
& -\left(S \delta_{i 1} \delta_{k 2}+2 \varepsilon_{i m k} \Omega_{m}\right) \overline{\theta u_{k}} \\
P= & -\overline{u_{i} u_{k}} \frac{\partial \overline{U_{i}}}{\partial x_{k}}-2 \varepsilon_{i m k} \Omega_{m} \overline{u_{i} u_{k}} \\
P_{\theta}= & -2 \overline{u_{i} \theta} \frac{\partial \bar{T}}{\partial x_{i}}
\end{aligned}
$$

Terms denoted by $\varepsilon$ are terms of dissipation due to molecular effects:

$$
\begin{aligned}
& \varepsilon_{i j}=2 v \overline{\frac{\partial u_{i}}{\partial x_{k}} \frac{\partial u_{j}}{\partial x_{k}}} \\
& \varepsilon_{i \theta}=(\alpha+v) \frac{\overline{\partial u_{i}} \frac{\partial \theta}{\partial x_{k}}}{\partial x_{k}} \\
& \varepsilon=v \frac{\partial \frac{\partial u_{i}}{\partial x_{k}} \frac{\partial u_{i}}{\partial x_{k}}}{\partial \theta} \frac{\partial \theta}{\partial x_{k}} \frac{\partial x_{k}}{\varepsilon_{\theta}}
\end{aligned}
$$

Finally, $\phi_{i j}$ and $\phi_{i \theta}$ are respectively terms of pressurestrain correlation and pressure-scalar gradient correlations:

$$
\begin{aligned}
& \left.\phi_{i j}=-\overline{\left(u_{j} \frac{\partial p}{\partial x_{i}}+u_{i} \frac{\partial p}{\partial x_{j}}\right.}\right) \\
& \phi_{i \theta}=-\overline{\theta \frac{\partial p}{\partial x_{i}}}
\end{aligned}
$$

In Cartesian notation, the evolution equations governing the considered flow are written as follows:

$$
\begin{aligned}
& \frac{d}{d t} \overline{u_{1}^{2}}=-2 S(1-2 R) \overline{u_{1} u_{2}}+\phi_{11}-\frac{2}{3} \varepsilon \\
& \frac{d}{d t} \overline{u_{2}^{2}}=-4 R S \overline{u_{1} u_{2}}+\phi_{22}-\frac{2}{3} \varepsilon \\
& \frac{d}{d t} \overline{u_{1} u_{2}}=-S(1-2 R) \overline{u_{2} u_{2}}-2 R S \overline{u_{1} u_{1}}+\phi_{12}
\end{aligned}
$$

$\frac{d k}{d t}=-\overline{u_{1} u_{2}} S-\varepsilon$

$\frac{d}{d t} \overline{\theta u_{1}}=-\overline{u_{1} u_{2}} G-S \overline{\theta u_{2}}+2 R \overline{\theta u_{2}}+\phi_{1 \theta}$

$\frac{d}{d t} \overline{\theta u_{2}}=-\overline{u_{2} u_{2}} G-2 R S \overline{\theta u_{1}}+\phi_{2 \theta}$

$\frac{d}{d t} \overline{\theta^{2}}=-2 \overline{\theta u_{2}} G-2 \varepsilon_{\theta}$

Where $\mathrm{R}=\Omega / \mathrm{S}$ is the non dimensional rotation number.

At this step of our work, we recall that the last general equations governing the flow will be integrated by two different methods. The first one is analytical and will be the object of the following section. The second one makes the object of sections 4 and 5 and consists on a numerical integration of time evolution equations when a second order modeling is retained.

\section{Analytical Solution When Non LINEAR EFFECTS ARE NEGLECTED}

In homogeneous sheared turbulence, Holt et al. (1992) pointed out in their results of direct numerical simulations that, at high shear, the effect of viscosity, pressure and non linear interactions are insignificant. If we take into account this hypothesis, we can considerably simplify the transport equations Eq. (8)Eq. (14) of turbulent parameters when non linear terms of pressure and viscosity are neglected. This leads to the following system of seven linear differential equations:

$\frac{d}{d t} \overline{u_{1}^{2}}=-2 S(1-2 R) \overline{u_{1} u_{2}}$

$\frac{d}{d t} \overline{u_{2}^{2}}=-4 R S \overline{u_{1} u_{2}}$

$\frac{d}{d t} \overline{u_{3}^{2}}=0$

$\frac{d}{d t} \overline{u_{1} u_{2}}=-S(1-2 R) \overline{u_{2} u_{2}}-2 R S \overline{u_{1} u_{1}}$

$\frac{d}{d t} \overline{\theta u_{1}}=-\overline{u_{2} u_{2}} G+(2 R-1) S \overline{\theta u_{2}}$

$\frac{d}{d t} \overline{\theta u_{2}}=-\overline{u_{2} u_{2}} G-2 R S \overline{\theta u_{1}}$

$\frac{d}{d t} \overline{\theta^{2}}=-2 \overline{\theta u_{2}} G$

It is a system of seven linear differential equations parameterized by the rotation number $(\mathrm{R}=\Omega / S)$ the ratio of the rotation rate to the shear rate. Solutions of such equations, at high shear, can be obtained by a simple analytical method. Laplace transformation can be used for solving the above system of seven differential equations.

$$
T L\{f(t)\}=\int_{0}^{\infty} f(t) e^{-p t} d t
$$

The application of Laplace transformation to the Eqs. (25)-(31) leads to the following solutions:

$$
\begin{gathered}
\overline{u_{1}^{2}}(\tau)=C_{11}+A_{11} \tau+D_{11} e^{4 R_{i} \tau} \\
\overline{u_{2}^{2}}(\tau)=C_{22}+A_{22} \tau+D_{22} e^{4 R_{i} \tau} \\
\overline{u_{3}^{2}}(\tau)=C_{33} \\
\overline{u_{1} u_{2}}(\tau)=C_{12}+D_{12} e^{4 R_{i} \tau} \\
\overline{\theta u_{1}}(\tau)=C_{1 \theta}+A_{1 \theta} \tau+B_{1 \theta} \tau^{2} \\
\quad+D_{1 \theta} e^{4 R_{i} \tau}+E_{1 \theta} e^{R_{i} \tau} \\
\overline{\theta u_{2}}(\tau)=C_{2 \theta}+A_{2 \theta} \tau+B_{1 \theta} \tau^{2} \\
\quad+D_{1 \theta} e^{4 R_{i} \tau}+E_{1 \theta} e^{R_{i} \tau} \\
\overline{\theta^{2}}(\tau)=C_{\theta \theta}+A_{\theta \theta} \tau+B_{\theta \theta} \tau^{2} \\
\quad+D_{\theta \theta} e^{4 R_{i} \tau}+E_{\theta \theta} e^{R_{i} \tau} \\
\overline{q^{2}}=\overline{u_{1}^{2}}+\overline{u_{2}^{2}}+\overline{u_{3}^{2}}
\end{gathered}
$$


Here $C, A, B, D$ and $E$ are functions of initial values of turbulent parameters and are parameterized by the Richardson number $\mathrm{R}_{\mathrm{i}}=2 \mathrm{R}(1-2 \mathrm{R})$ (see appendix $\mathrm{A}$ for the complete expressions of these coefficients). The non dimensional time is defined by $\tau=S t$.

We point out that the same hypothesis has been retained in our previous work Bouzaiane et al. (2003), and analogous solutions are derived for the case of the stably stratified homogeneous turbulence. Solutions were parameterized by the analogous non dimensional Richardson number of gradient. Solutions (32)-(39) will be now investigated to confirm the existence of asymptotic behavior (when $\tau \rightarrow+\infty$ ) of dimensionless kinematic and scalar parameters. We can deduce firstly the expressions of the anisotropic components $b_{11}, b_{22}, b_{33}, b_{12}$ from the above solutions where $b_{i j}=\frac{\overline{u_{i} u_{j}}}{2 K}-\frac{\delta_{i j}}{3}$ are components of the anisotropic tensor b Schiestel (1997):

$$
\begin{aligned}
b_{11} & =\frac{\overline{u_{1}^{2}}}{\overline{q^{2}}}-\frac{1}{3} \\
& =\frac{C_{11}+A_{11} \tau+D_{11} e^{4 R_{i} \tau}}{C_{q}+A_{q} \tau+D_{q} e^{4 R_{i} \tau}}-\frac{1}{3} \\
b_{22} & =\frac{\overline{u_{2}^{2}}}{\overline{q^{2}}}-\frac{1}{3} \\
& =\frac{C_{22}+A_{22} \tau+D_{22} e^{4 R_{i} \tau}}{C_{q}+A_{q} \tau+D_{q} e^{4 R_{i} \tau}}-\frac{1}{3} \\
b_{33} & =\frac{\overline{u_{3}^{2}}}{\overline{q^{2}}}-\frac{1}{3} \\
& =\frac{C_{33}}{C_{q}+A_{q} \tau+D_{q} e^{4 R_{i} \tau}}-\frac{1}{3} \\
b_{12} & =\frac{\frac{u_{1} u_{2}}{q^{2}}}{} \\
& =\frac{C_{12}+D_{12} e^{4 R_{i} \tau}}{C_{q}+A_{q} \tau+D_{q} e^{4 R_{i} \tau}}
\end{aligned}
$$

At long time evolution (while $(\tau=S t) \rightarrow+\infty)$, we obtain:

$$
\begin{aligned}
& \left(b_{11}\right)_{\infty}=\frac{D_{11}}{D_{q}}-\frac{1}{3} \\
& \left(b_{22}\right)_{\infty}=\frac{D_{22}}{D_{q}}-\frac{1}{3} \\
& \left(b_{33}\right)_{\infty}=-\frac{1}{3} \\
& \left(b_{12}\right)_{\infty}=\frac{D_{12}}{D_{q}}
\end{aligned}
$$

In an analogous manner, we can deduce from solutions (34)-(37) the analytic expression of scalar nondimensional parameters $\frac{\overline{\theta u_{1}}}{\overline{\theta u_{2}}}, \frac{\overline{\theta u_{1}}}{\theta^{\prime} u_{1}^{\prime}}, \frac{\theta^{\prime} / G}{q^{\prime} / S}$ where $\theta^{\prime}=\sqrt{\overline{\theta^{2}}}$ is the root mean square of the scalar fluctuations and $q^{\prime}=\sqrt{\overline{q^{2}}}$ where $\overline{q^{2}}=2 K$.

$$
\begin{aligned}
& \frac{\overline{\theta u_{1}}}{\overline{\theta u_{2}}} \simeq \frac{D_{1 \theta} e^{4 R_{i} \tau}}{D_{2 \theta} e^{4 R_{i} \tau}} \\
& \frac{\overline{\theta u_{1}}}{\theta^{\prime} u_{1}^{\prime}} \simeq \frac{D_{1 \theta} e^{4 R_{i} \tau}}{D_{11}^{1 / 2} D_{\theta \theta}^{1 / 2} e^{4 R_{i} \tau}} \\
& \frac{\theta^{\prime} / G}{q^{\prime} / S} \simeq \frac{1}{\alpha} \frac{D_{\theta \theta}^{1 / 2} e^{2 R_{i} \tau}}{D_{q}^{1 / 2} e^{2 R_{i} \tau}}
\end{aligned}
$$

When $\tau \rightarrow+\infty$, we obtain the asymptotic equilibrium states for $\frac{\overline{\theta u_{1}}}{\overline{\theta u_{2}}}, \frac{\overline{\theta u_{1}}}{\theta^{\prime} u_{1}^{\prime}}$ and $\frac{\theta^{\prime} / G}{q^{\prime} / S}$

$$
\begin{aligned}
& \left(\frac{\overline{\theta u_{1}}}{\overline{\theta u_{2}}}\right)_{\infty}=\frac{D_{1 \theta}}{D_{2 \theta}} \\
& \left(\frac{\overline{\theta u_{1}}}{\theta^{\prime} u_{1}^{\prime}}\right)_{\infty}=\frac{D_{1 \theta}}{D_{11}^{1 / 2} D_{\theta \theta}^{1 / 2}} \\
& \left(\frac{\theta^{\prime} / G}{q^{\prime} / S}\right)_{\infty}=\frac{1}{\alpha} \frac{D_{\theta \theta}^{1 / 2}}{D_{q}^{1 / 2}}
\end{aligned}
$$

The obtained expressions of $b_{11}, b_{22}, b_{33}, b_{12} \frac{\overline{\theta u_{1}}}{\overline{\theta u_{2}}}, \frac{\overline{\theta u_{1}}}{\theta^{\prime} u_{1}^{\prime}}, \frac{\theta^{\prime} / G}{q^{\prime} / S} \quad$ are constants depending only on the Richardson number $R_{i}$ and initial values of turbulent parameters. These results confirm the existence of equilibrium states for these kinematic and scalars parameters, at long time evolution. This first approach is only a qualitative one and has confirmed at long time evolutions (when $\tau \rightarrow+\infty$ ), the existence of an asymptotic equilibrium behavior for kinematic and scalar dimensionless parameters. In the following section a quantitative study of the asymptotic equilibrium behavior of the above mentioned dimensionless parameters is conducted when a second order modeling is retained.

\section{SECOND ORDER MODELING}

In this part of our work, second order closure models are retained to close transport equations Eqs. (18)-(24). The pressure-strain correlation $\phi_{i j}$ and pressure-scalar gradient correlations are the principal terms to be modeled in evolution equations of Reynolds stress and turbulent scalar flux. These correlations $\phi_{i j}$ and $\phi_{i \theta}$ are classically decomposed on two contributions (Cadiou 1996):

$$
\begin{aligned}
& \phi_{i j}=\phi_{i j}^{1}+\phi_{i j}^{2} \\
& \phi_{i \theta}=\phi_{i \theta}^{1}+\phi_{i \theta}^{2}
\end{aligned}
$$

The first contributions are called slow terms or the return-to-the isotropy terms and they characterize the non linear mechanism of interaction between turbulent fluctuations. The second ones are usually called rapid terms and they represent the interaction between mean and turbulent flows. Since there is not a universal model that predict correctly all turbulent flows Hanjaclic (1994) and Sodja (2007), three of the most 
used second order models are retained and will be presented in the following subsections.

\subsection{The Launder, Reece and Rodi Models}

The Launder, Reece and Rodi (LRR) (Launder et al. 1975) models for the pressure-strain and pressure-scalar gradient correlations are the most popular models used by authors. These models submitted only to the general kinematic constraints take the following forms:

$$
\begin{aligned}
\phi_{i j}^{1}= & -C_{1} \varepsilon b_{i j} \\
\phi_{i j}^{2}= & C_{2}\left(\overline{u_{i} u_{k}} \widetilde{U}_{j, k}+\overline{u_{i} u_{k}} \widetilde{U}_{k, j}\right. \\
& \left.-\frac{2}{3} C_{2} \overline{u_{m} u_{n}} \widetilde{U}_{m, n} \delta_{i j}\right)
\end{aligned}
$$

Here $\widetilde{U}_{k, j}=\bar{U}_{k, j}+\varepsilon_{k j m} \Omega_{m}$

(where $\bar{U}_{i, k}$ is the derivative $\frac{\partial \bar{U}_{i}}{\partial x_{k}}$ )

where $C_{1}=1.8$ and $\mathrm{C}_{2}=0.6$. and $\delta_{i j}$ are components of identity tensor,

For the pressure-scalar gradient correlation, slow and rapid terms are written in the following form:

$$
\phi_{i \theta}^{1}=-C_{1}^{\prime} \frac{\varepsilon}{k} \overline{\theta u_{i}}
$$

and

$$
\begin{aligned}
\phi_{i \theta}^{2}= & 0,8 \overline{\theta u}_{k} \bar{U}_{i, k}-0,2 \overline{\theta u}_{k} \bar{U}_{k, i} \\
& +R \overline{\theta u}_{k} \varepsilon_{i j k}\left(\bar{U}_{i, k}+\bar{U}_{k, i}\right)
\end{aligned}
$$

Where $C_{1}^{\prime}=3.2$

The Launder Reece Rodi model will be called in the present work model 1 .

\subsection{The Speziale, Sarkar and Gatski Model}

The Speziale, Sarkar et Gatski (SSG) second order closure model has know a great success during the last two decades. It has been submitted in addition to the kinematics constraints to the strong form of realisability. Furthermore it predicts stable fixed points (Speziale et al. 1990). The final form of this model is the followed one:

$$
\begin{aligned}
& \phi_{i j}=-3.4 \varepsilon b_{i j}+4.2\left(b_{i k} b_{k j}-\frac{1}{3} b_{k l} b_{k l} \delta_{i j}\right) \\
& +4.15 k \bar{S}_{i j}+1.25 k\left(b_{i k} \bar{S}_{j k}+b_{j k} \bar{S}_{i k}\right. \\
& \left.-\frac{2}{3} b_{k l} \bar{S}_{k l} \delta_{i j}\right)+0.4 k\left(b_{i k} \widetilde{w}_{j k}+b_{j k} \tilde{w}_{i k}\right)
\end{aligned}
$$

$\bar{S}_{i j}$ are the components of the symmetric part of gra $\overline{\bar{U}}$ :

$\bar{S}_{i j}=\frac{1}{2}\left(\frac{\partial \overline{U_{i}}}{\partial x_{j}}+\frac{\partial \overline{U_{j}}}{\partial x_{i}}\right)$,

$\bar{W}_{i j}$ are the components of its ant symmetric part

$$
\bar{W}_{i j}=\frac{1}{2}\left(\frac{\partial \overline{U_{i}}}{\partial x_{j}}-\frac{\partial \overline{U_{j}}}{\partial x_{i}}\right)
$$

And:

$$
\widetilde{W}_{j, k}=\bar{W}_{j, k}+\varepsilon_{3 k j} \Omega
$$

We point out here that this model has not been, to our knowledge, extended to the to the pressure scalar gradient correlation. However to obtain a closed system of equations, the SSG model will be coupled with the LRR model of pressure scalar gradient. This coupling will be called model 2 .

\subsection{The Shih and Lumley Models}

This model has been submitted also in addition to the general kinematics constraints to the strong form of realisability (Shih et al. 1985 and Ristrocelli et al. 1988). This model constitutes a complete class of model for both kinematic and scalar effects. The model of pressure-strain correlation of Shih and Lumley (Shih 1996) is written on the following form.

$$
\begin{aligned}
& \phi_{i j}=-\beta \varepsilon_{i j} b_{i j}+\left(\frac{4}{5}+8 \alpha_{5}\right) \bar{S}_{i j}-\frac{2}{3}\left(1-\alpha_{5}\right) \\
& \left(\tau_{i k} \widetilde{U}_{j, k}+\tau_{j k} \widetilde{U}_{i, k}\right)-\frac{2}{3} \tau_{i j} \bar{S}_{i j} \delta_{i j} \\
& +\frac{2}{3}\left(1+8 \alpha_{5}\right)\left(\tau_{i k} \widetilde{U}_{j, k}+\tau_{j k} \widetilde{U}_{i, k}-\frac{2}{3} \tau_{i j} \bar{S}_{i j} \delta_{i j}\right) \\
& +\frac{6}{5} \tau_{i j} \bar{S}_{i j} b_{i j}+\frac{4}{15}\left(\tau_{i j} \widetilde{W}_{i j}+\tau_{j i} \widetilde{W}_{j i}\right)+\frac{5}{K} \\
& \left(\tau_{i l} \tau_{l k} \widetilde{U}_{j, k}+\tau_{j l} \tau_{l k} \widetilde{U}_{i, k}-2 \tau_{i l} \tau_{j k} \bar{S}_{l k}\right)
\end{aligned}
$$

Here $\tau_{i j}-\overline{u_{i} u_{j}}$

The return-to-isotropy term, and linear terms of pressure scalar gradient correlation are written as

$$
\begin{aligned}
\phi_{i \theta}^{1} & =-\frac{\varepsilon}{\overline{q^{2}}} \phi^{\theta} \overline{\theta u}_{i} \\
\phi_{i \theta}^{2} & =2 \bar{U}_{j, k} I_{i j k}
\end{aligned}
$$

Whereas detailed expressions of $\phi_{\theta}$ and $\mathrm{I}_{\mathrm{ijk}}$ (Shih et al. 1996) are presented in appendix B. The Shih Lumley model will be called here model 3.

\section{Modeling EQUATIONS EQUILIBRIUM STATES}

\subsection{Modeling Equations by Model 1}

The Launder Reece and Rodi models for pressure-strain and pressure-scalar correlations are introduced in evolution Eqs. (18)-(24) which are associated to modeled transport equations of the turbulent kinetic energy dissipation and scalar variance dissipation. The following differential system of equations is thus obtained:

$$
\begin{aligned}
\frac{d \bar{u}_{1}^{2}}{d t}= & -2 S(1-2 R) \overline{u_{1} u_{2}}-C_{1} \varepsilon b_{11} \\
& +C_{2}\left(1-\frac{2}{3} C_{2}\right) \overline{u_{1} u_{2}} S-\frac{2}{3} \varepsilon \\
\frac{d \bar{u}_{2}^{2}}{d t}= & -4 R S \overline{u_{1} u_{2}}-C_{1} \varepsilon b_{22} \\
& +C_{2}\left(1-\frac{2}{3} C_{2}\right) \overline{u_{1} u_{2}} S-\frac{2}{3} \varepsilon
\end{aligned}
$$




$$
\begin{aligned}
\frac{d \overline{u_{1} u_{2}}}{d t}= & -S(1-2 R) \overline{u_{2}^{2}}-2 R S \overline{u_{1}^{2}} \\
\frac{d K}{d t}= & -C_{1} \varepsilon b_{12}+C_{2} \overline{u_{1}^{2}} S \\
\frac{d \varepsilon}{d t}= & -C_{\varepsilon 1} \frac{\varepsilon}{K} \overline{u_{1} u_{2} S}-C_{\varepsilon 2} \frac{\varepsilon^{2}}{K} \\
\frac{d \overline{\theta u_{1}}}{d t}= & -\overline{u_{1} u_{2}} G-1.8 S \overline{\theta u_{2}}+3 R S \overline{\theta u_{2}} \\
& -C_{1}^{\prime} \frac{\varepsilon}{K} \overline{\theta u_{1}} \\
\frac{d \overline{\theta u_{2}}}{d t}= & -\overline{u_{2} u_{2}} G+0.2 S \overline{\theta u_{1}}-3 R S \overline{\theta u_{1}} \\
& -C_{1}^{\prime} \frac{\varepsilon}{K} \overline{\theta u_{2}} \\
\frac{d \overline{\theta^{2}}}{d t}= & -2 \overline{\theta u_{2}} G-2 \varepsilon_{\theta} \\
\frac{d \varepsilon_{\theta}}{d t}= & -C_{d 1} \frac{\varepsilon_{\theta}^{2}}{\theta^{2}}-2 C_{d 2} \frac{\varepsilon}{K} \varepsilon_{\theta}-C_{d 3} \frac{\varepsilon}{K} \overline{\theta u_{2}} G \\
& -2 C_{d 4} \frac{\varepsilon_{\theta}}{K} \overline{u_{1} u_{2} S}
\end{aligned}
$$

Two others non linear differential systems are obtained when model 2 in one hand and model 3 in another hand are introduced in the general Eqs. (18)-(24).

\subsection{Non Dimensional Equations}

The previous differential equations are castled in non dimensional forms when non dimensional parameters $\mathrm{b}_{11}, \mathrm{~b}_{22}, \mathrm{~b}_{12}$ and $\frac{\varepsilon}{K S}$ are introduced for the kinematic field and the ratio of scalar fluxes $\rho_{1}=\frac{\overline{\theta u_{1}}}{\overline{\theta u_{2}}}$, the turbulent scalar correlation coefficient $\rho_{2}=\frac{\overline{\theta u_{1}}}{\theta^{\prime} u_{1}^{\prime}}$ and the ratio $\rho_{3}=\frac{\theta^{\prime} / G}{q^{\prime} / S}$ introduced for the scalar field.

In this section, all non dimensional parameters substitute previous turbulent ones $\overline{u_{i} u_{j}}, \overline{u_{i} \theta}, K, \varepsilon$ and $\overline{\theta^{2}}$.

Non dimensional equations modeled by the classic model 1 are given by:

$$
\begin{aligned}
\frac{d b_{11}}{d \tau} & =-2(1-2 R) b_{12}-\frac{C_{1}}{2} \frac{\varepsilon}{K S} b_{11} \\
C_{2} & \left(1-\frac{2}{3} C_{2}\right) b_{12}-\frac{1}{3} \frac{\varepsilon}{K S}+2 b_{12}\left(b_{11}+\frac{1}{3}\right) \\
& -\left(b_{11}+\frac{1}{3}\right) \frac{\varepsilon}{K S} \\
\frac{d b_{22}}{d \tau} & =-4 R b_{12}-\frac{C_{1}}{2} \frac{\varepsilon}{K S} b_{22}+C_{2}\left(1-\frac{2}{3} C_{2}\right) b_{12} \\
& -\frac{1}{3} \frac{\varepsilon}{K S}+2 b_{12}\left(b_{22}+\frac{1}{3}\right)
\end{aligned}
$$

$$
\begin{aligned}
& +\left(b_{22}+\frac{1}{3}\right) \frac{\varepsilon}{K S} \\
& \frac{d b_{12}}{d \tau}=(2 R-1)\left(b_{22}+\frac{1}{3}\right)-2 R\left(b_{11}+\frac{1}{3}\right) \\
& -\frac{C_{1}}{2} \frac{\varepsilon}{K S} b_{12}+C_{2}\left(b_{11}+\frac{1}{3}\right) \\
& +2 b_{12}^{2}+\frac{\varepsilon}{K S} b_{12} \\
& \frac{d}{d \tau}\left(\frac{\varepsilon}{K S}\right)=2\left(1-C_{\varepsilon 1}\right) \frac{\varepsilon}{K S} b_{12} \\
& +\left(1-C_{\varepsilon 2}\right)\left(\frac{\varepsilon}{K S}\right)^{2} \\
& \frac{d \rho_{1}}{d \tau}=-\frac{\rho_{1} \rho_{3}}{\rho_{2}} \frac{b_{12}}{\left(b_{11}+\frac{1}{3}\right)^{0.5}}+3 R-1.8 \\
& +\frac{\rho_{1}^{2} \rho_{3}}{\rho_{2}} \frac{b_{22}+\frac{1}{3}}{\left(b_{11}+\frac{1}{3}\right)^{0.5}}+(3 R-0.2) \rho_{1}^{2} \\
& \frac{d \rho_{2}}{d \tau}=-\frac{1}{\rho_{3}} \frac{b_{12}}{\left(b_{11}+\frac{1}{3}\right)^{0.5}}-1.8 \rho_{1} \rho_{2} \\
& +3 R \rho_{1} \rho_{2}-C_{1}^{\prime} \frac{\varepsilon}{K S} \rho_{2}+(1-2 R) \rho_{1} \frac{b_{12}}{b_{11}+\frac{1}{3}} \\
& +\frac{C_{1}}{4} \rho_{2} \frac{\varepsilon}{K S} \frac{b_{11}}{b_{11}+\frac{1}{3}}-\frac{C_{2}}{2}\left(1-\frac{2}{3} C_{2}\right) \rho_{2} \frac{b_{12}}{b_{11}+\frac{1}{3}} \\
& +\frac{1}{6} \rho_{2} \frac{\varepsilon}{K S} \frac{1}{b_{11}+\frac{1}{3}}+\frac{\rho_{1} \rho_{2}^{2}}{\rho_{3}}\left(b_{11}+\frac{1}{3}\right)^{0.5} \\
& +\frac{r_{C}}{2} \rho_{2} \frac{\varepsilon}{K S} \\
& \frac{d \rho_{3}}{d \tau}=-\rho_{1} \rho_{2}\left(b_{11}+\frac{1}{3}\right)^{0.5}-\frac{r_{C}}{2} \rho_{3} \frac{\varepsilon}{K S} \\
& +\rho_{3} b_{12}+\frac{1}{2} \rho_{3} \frac{\varepsilon}{K S}
\end{aligned}
$$

In the Eqs. (85) and (86) $r_{c}$ is the characteristic time rate

$$
r_{c}=\frac{\overline{q^{2}} / \varepsilon}{\overline{\theta^{2}} / \varepsilon_{\theta}}
$$

\subsection{Numerical integration and results}

\subsubsection{Asymptotic Equilibrium States}

Since wealth of numerical simulations and experimental results has shown that at long time evolution, non dimensional parameters have a general tendency to asymptotic equilibrium states, peculiar attention will be accorded here to such behavior. The three non linear differential equations are submitted to the initial isotropic conditions of the Direct Numerical Simulation (DNS) of Brethouwer (2005) for the kinematic field. The initial condition $\left(\rho_{1}\right)_{0}=-1.86,\left(\rho_{2}\right)_{0}=0.43$, and $\left.\left(\rho_{3}\right)_{0}=0.74\right)$ of the experimental results of Tavoularis and Corrsin (1981), initially isotropic also for the 
kinematic field, are retained for the scalar field. The fourth Runge-Kutta method is used to integrate the three non linear differential systems obtained after modeling. The numerical integration is advanced to long time evolution ( $\tau=\mathrm{St}=20$ ) and is carried out separately for the values $0,0.25,0.5,-0.25,-0.5$ and 0.75 of the dimensionless rotation number $R$ (the ratio of the rotation rate to the shear rate). At long time evolution a general tendency to asymptotic equilibrium states for the non dimensional parameters has been observed. Obtained asymptotic equilibrium states of kinematic parameters (the components $b_{11}, b_{22}, b_{12}$ of anisotropic tensor and the non dimensional shear number $\varepsilon / K S$ ) and scalar non dimensional parameters: the turbulent scalar flux rate $\rho_{1}=\frac{\overline{\theta u_{1}}}{\overline{\theta u_{2}}}$, the correlation coefficient $\rho_{2}=\frac{\overline{\theta u_{1}}}{\theta^{\prime} u_{1}^{\prime}}$ and the ratio $\rho_{3}=\frac{\theta^{\prime} / G}{q^{\prime} / S}$ are summarized on Tables 1 and 2.

Table 1 Equilibrium values predicted by kinematic parameters

\begin{tabular}{|c|c|c|c|c|}
\hline \multicolumn{2}{|c|}{} & $\mathrm{R}=0$ & $\mathrm{R}=0.25$ & $\mathrm{R}=0.5$ \\
\hline \multirow{4}{*}{$\begin{array}{c}\text { Model } \\
1\end{array}$} & $\mathrm{~b}_{11}$ & 0.192 & -0.059 & - \\
\cline { 2 - 5 } & $\mathrm{b}_{22}$ & -0.095 & 0.155 & - \\
\cline { 2 - 5 } & $\mathrm{b}_{12}$ & -0.185 & -0.207 & - \\
\cline { 2 - 5 } & $\varepsilon / \mathrm{KS}$ & 0.182 & 0.206 & 0.019 \\
\hline \multirow{4}{*}{$\begin{array}{c}\text { Model } \\
2\end{array}$} & $\mathrm{~b}_{11}$ & 0.220 & 0.537 & 1 \\
\cline { 2 - 5 } & $\mathrm{b}_{22}$ & -0.147 & -0.201 & -0.320 \\
\cline { 2 - 5 } & $\mathrm{b}_{12}$ & -0.164 & -0.365 & -0.689 \\
\cline { 2 - 5 } Model & $\varepsilon / \mathrm{KS}$ & 0.167 & 0.387 & 0.730 \\
\hline \multirow{4}{*}{3} & $\mathrm{~b}_{11}$ & 0.114 & -0.069 & -0.123 \\
\cline { 2 - 5 } & $\mathrm{b}_{22}$ & -0.116 & 0.067 & 0.120 \\
\cline { 2 - 5 } & $\mathrm{b}_{12}$ & -0.121 & -0.152 & -0.004 \\
\cline { 2 - 5 } & $\varepsilon / \mathrm{KS}$ & 0.144 & 0.181 & 0.014 \\
\hline \multirow{4}{*}{ DNS } & $\mathrm{b}_{11}$ & 0.353 & 0.200 & - \\
\cline { 2 - 5 } & $\mathrm{b}_{22}$ & -0.112 & 0.031 & - \\
\cline { 2 - 5 } & $\mathrm{b}_{12}$ & -0.125 & 0 & - \\
\cline { 2 - 5 } & $\varepsilon / \mathrm{KS}$ & - & - & - \\
\hline
\end{tabular}

Table 2 Equilibrium values predicted for scalar parameters

\begin{tabular}{|c|c|c|c|c|}
\hline \multicolumn{2}{|c|}{} & $\mathrm{R}=0$ & $\mathrm{R}=0.25$ & $\mathrm{R}=0.5$ \\
\hline \multirow{4}{*}{$\begin{array}{c}\text { Model } \\
1\end{array}$} & $\rho_{1}$ & -1.08 & -0.283 & - \\
\cline { 2 - 5 } & $\rho_{2}$ & 0.963 & 0.461 & - \\
\cline { 2 - 5 } & $\rho_{3}$ & 1.41 & 1.64 & - \\
\hline \multirow{3}{*}{$\begin{array}{c}\text { Model } \\
2\end{array}$} & $\rho_{1}$ & -1.12 & -1.46 & -1.8 \\
\cline { 2 - 5 } & $\rho_{2}$ & 0.297 & 0.621 & - \\
\cline { 2 - 5 } & $\rho_{3}$ & 2.44 & 2.27 & - \\
\hline \multirow{3}{*}{$\begin{array}{c}\text { Model } \\
3\end{array}$} & $\rho_{1}$ & -0.865 & -0.240 & 5.44 \\
\cline { 2 - 5 } & $\rho_{2}$ & 0.616 & 0.311 & -0.842 \\
\cline { 2 - 5 } DNS & $\rho_{3}$ & 0.834 & 0.930 & - \\
\hline \multirow{3}{*}{} & $\rho_{1}$ & - & - & - \\
\cline { 2 - 5 } & $\rho_{2}$ & 0.800 & 0.472 & - \\
\cline { 2 - 5 } & $\rho_{3}$ & 0.846 & 1.230 & - \\
\hline
\end{tabular}

It is essential here to note that asymptotic equilibrium values obtained for the kinematic field are with a slight difference equal to equilibrium state values obtained by Sarkar and Spezial (1989).

Asymptotic equilibrium states have been obtained for different cases. An exception is observed for the LRR model for the value $\mathrm{R}=0.5$ of the non dimensional rotation number where no tendency to equilibrium states has been observed. An improve in predicting equilibrium states is observed for model 2 where the SSG model is retained for the pressure strain correlation. In fact, the equilibrium values predicted by the model 2 have been found for non dimensional rotation rates in the larger interval $-0.75 \leq \mathrm{R} \leq 0.5$ whereas the model 1 and model 3 predict equilibrium states for only positive values of non dimensional rotation number. The model 1 has predicted asymptotic equilibrium states only for $\mathrm{R}=0.25$ for both kinematic and scalar fields. Model 3 has predicted an equilibrium states for the two values $\mathrm{R}=0.25$ and $\mathrm{R}=0.5$ of the rotation number $\mathrm{R}$ for both kinematic and scalar fields.

In comparison to values of the DNS of Brethouwer for which asymptotic equilibrium states are obtained for the value 0.25 of the rotation number, model 1 and model 3 show the best agreement for the dimensionless parameters $\rho_{2}$ and $\rho_{3}$. Whereas model 2 under estimates the values of the DNS of Brethouwer for the dimensionless ratio $\rho_{2}$. Model 2 estimates to $20 \%$ the values of DNS whereas model 3 under estimates this last value to $25 \%$ approximately. For the ratio $\rho_{3}$, model 1 shows an under estimation of $25 \%$ values of DNS whereas the model 3 indicates an over estimation of 25 $\%$ this last value. Model 2 shows an under estimation of $50 \%$ the value obtained by DNS for $\rho_{2}$ and an under estimation of $100 \%$ for the ratio $\rho_{3}$ compared to model 1 . A better agreement is observed by both model 2 and model 3 . The acceptable agreement results obtained by models 2 and 3, compared to model 1 , can be justified if we precise that these models are submitted to the strict conditions of realizability Shih et al. (1985a,b, 1996), whereas model 1 has been submitted only to the known relations of kinematic constraints Lumley (1985). Rotation affects not only the kinematic fields by increasing the anisotropy but also the scalar field. It is the turbulent flux rate that is the most concerned by the effects rotation.

\subsubsection{Evolutions of Non Dimensional Parameters}

In this subsection time evolutions of non dimensional parameters versus non dimensional time for different rotation numbers are addressed. The kinematic field is firstly analyzed. Evolutions of the turbulent kinetic energy are discussed. Then the evolution of non dimensional rates for the scalar field are explained. The time development of the turbulent kinetic energy is presented in Figs. 2, 3 and 4. All these evolutions start from the initial value $(\mathrm{K})_{0}=0.33 \mathrm{~m}^{2} \mathrm{~s}^{-2}$. Evolutions of the turbulent kinetic energy exhibit the same behavior. They show an exponential growth of the turbulent kinetic energy for different values of non dimensional rotation number. A strong influence of rotation on the stability of the flow is clearly observed on these figures. 
In Fig. 2 time evolutions of turbulent kinetic energy according to the three models for the cases $\mathrm{R}=0$ and $\mathrm{R}=0.25$ of non dimensional rotation number $\mathrm{R}$ are displayed.

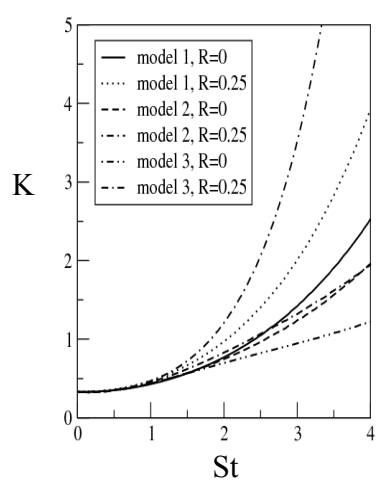

Fig. 2. Time evolutions of the turbulent kinetic energy

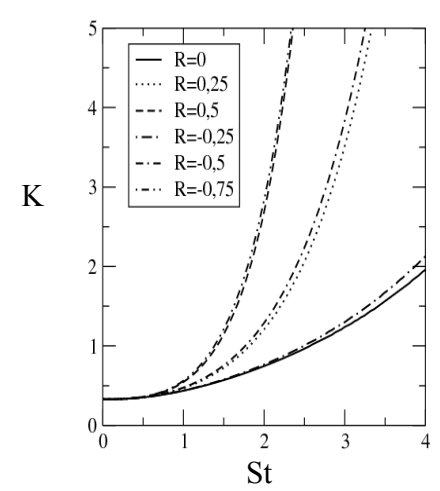

Fig. 3. Time evolutions of the turbulent kinetic energy for various values of non dimensional rotation number according to the model 2 .

A weak growth of turbulent kinetic energy is observed by the model 1 for $\mathrm{R}=0$ and $\mathrm{R}=0.25$, a moderate growth is observed by the model 3 and the strongest growth is observed by the model 2 . On Fig. 3 only the time evolutions of turbulent kinetic energy according to the model 2 are plotted for six different rotation number. The turbulent kinetic energy has a very fast exponential growth, at larger values of non dimensional time St, which is qualitatively in agreement with recent results of Brethouwer.

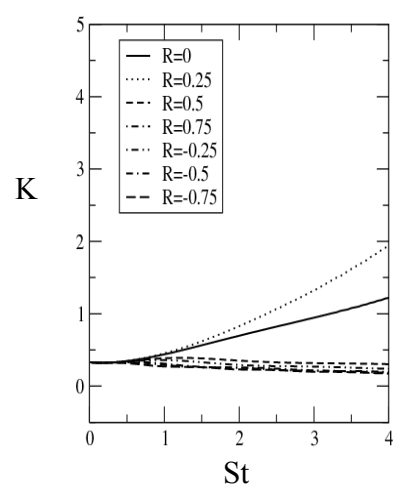

Fig. 4. Time evolutions of the turbulent kinetic energy for various cases of non dimensional rotation number according to the model 3 .
Figure 4 shows time evolution of the turbulent kinetic energy according to the model 3 for several values of non dimensional rotation number $\mathrm{R}$. Both non rotating case and the case of $\mathrm{R}=0.25$ show an exponential growth of turbulent kinetic energy. For all other cases $\mathrm{R}=0.5, \mathrm{R}=0.75, \mathrm{R}=-0.25, \mathrm{R}=-0.5$ and $\mathrm{R}=-0.75$ the turbulent kinetic energy lead to a decay and this is also in agreement with previous results Brethouwer (2005), Jacobitz et al. (2008).

Figures 5 and 6 show time evolution of the turbulent scalar fluxes ratio according to three models for the values $\mathrm{R}=0$ and $\mathrm{R}=0.25$ of non dimensional rotation number.

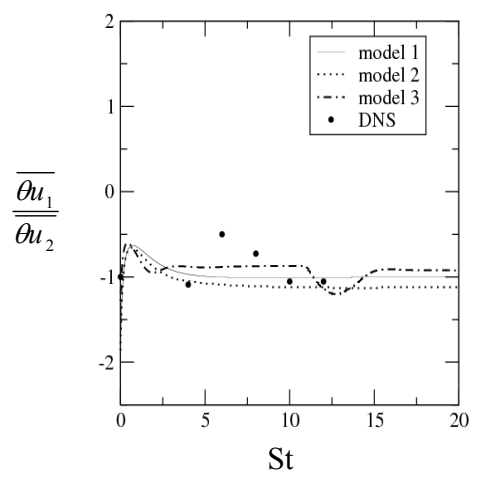

Fig. 5. Time evolutions of the turbulent scalar fluxes ratio for three models in the case $\mathrm{R}=0$.

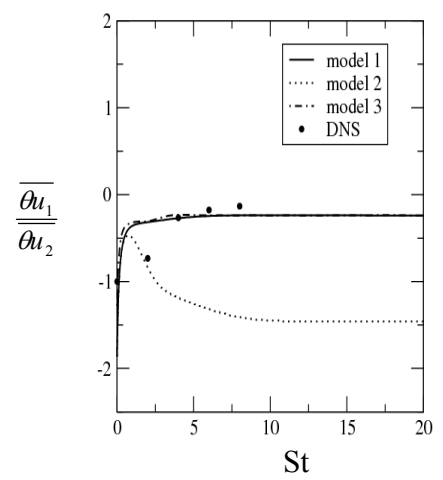

Fig. 6. Time evolutions of the turbulent scalar fluxes ratio for three models in the case $\mathrm{R}=0.25$.

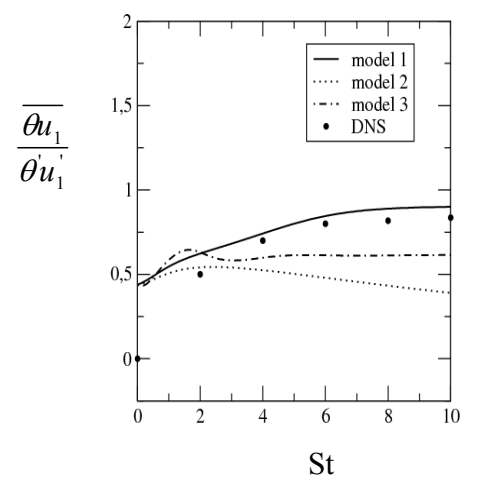

Fig. 7. Time evolutions of the correlation coefficient for three models in the case $\mathrm{R}=0$.

On these figures the asymptotic equilibrium behavior is observed for each of three models. In the case $\mathrm{R}=0$, a perfect agreement of model 1 and model 2 with the recent results of Brethouwer are clearly observed for 
St $>10$ but in the case $\mathrm{R}=0.25$ the agreement with DNS results of Brethouwer is observed on the evolution of model 1 and model 3 only for $\mathrm{St}>5$.

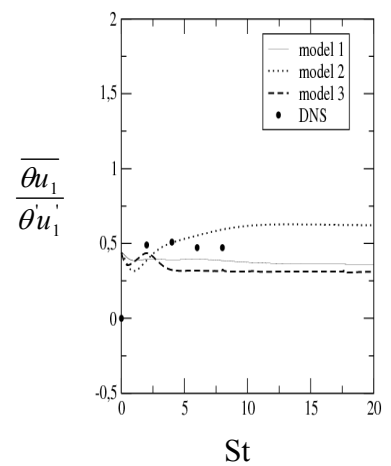

Fig. 8. Time evolutions of the correlation coefficient for three models in the case $\mathrm{R}=0.25$.

On Figs. 7 and 8, time evolutions of correlation coefficient are plotted according to three models for the values $\mathrm{R}=0$ and $\mathrm{R}=0.25$ of non dimensional rotation number. The agreement of model 1 with recent results of Brethouwer is noted for $\mathrm{St}>4$ in the case $\mathrm{R}=0$ and for $\mathrm{St}>5$ in the case $\mathrm{R}=0.25$. An agreement between predictions of model 1 and model 2 is obtained in this last case. On Figs. 9 and 10, time evolutions of the ratio $\rho_{3}=\frac{\theta^{\prime} / G}{q^{\prime} / S}$ are shown.

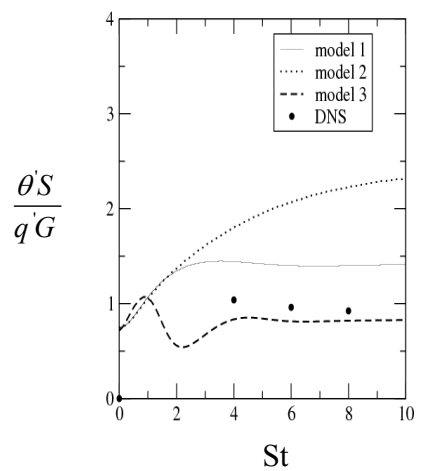

Fig. 9. Time evolutions of the ratio $\frac{\theta^{\prime} S}{q^{\prime} G}$ for three models in the case $\mathrm{R}=0$.

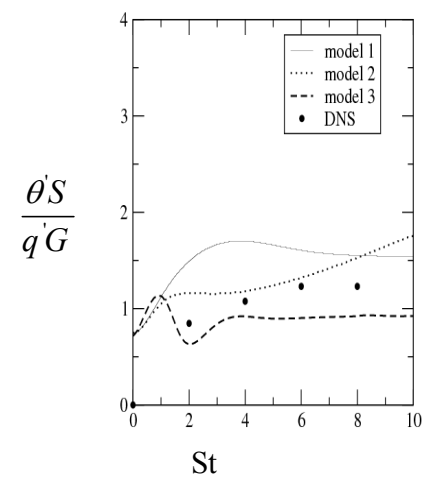

Fig. 10. Time evolutions of the ratio $\frac{\theta \text { 'S }}{q^{\prime} G}$ for three models in the case $\mathrm{R}=0.25$.

On these figures, the asymptotic equilibrium behavior is clearly observed by the predictions of model 1 and model 3. On Fig. 9, the agreement of the model 3 with recent results of Brethouwer is clearly observed for $\mathrm{St}>4$. whereas on Fig. 10 the concordance is noted between model 2 and DNS results of Brethouwer for $3<\mathrm{St}<7$.

\section{CONCLUSions}

In this study, the effects of rotation on kinematic and scalar fields of two dimensional homogeneous sheared turbulence are studied through two different methods. On the first method, the asymptotic equilibrium behaviors are confirmed by analytical solutions of equations describing the considered flow. These analytical solutions, available at strong shear and when non linear effects are neglected and have been obtained by Laplace transformation applied to seven linear differential equations. The second method is numerical and based on a second order modeling of time evolution equations. Three different models between the most known ones have been retained for the problem of homogeneous turbulent shear flow submitted to the rotation and superimposed to a passive scalar field with a constant mean gradient. Retained models are denoted respectively here model 1 , model 2 and model 3 . We are interested essentially to the prediction of asymptotic equilibrium states of dimensionless parameters characterizing the kinematic and scalar fields. A non dimensional form of equations is obtained when non dimensional kinematic and scalar dimensionless parameters are introduced. The numerical integration has been conducted by the fourth order Runge-Kutta method for several values of rotation number $\mathrm{R}$ and is advanced to a sufficiently long time evolution. This second approach has shown, in the most cases, that the predictions of retained second order models confirm the existence of the asymptotic equilibrium states. The following definitive conclusions can be drawn:

The model 1 has predicted equilibrium states whereas the model 3 has shown better predictions than the model 1 compared to DNS values. The model 2 has shown the acceptable prediction of equilibrium states. Furthermore, compared to model 1, the model 2 has been of a great contribution on predicting asymptotic equilibrium values of the scalar field. For the turbulent kinetic energy, an exponential growth with the non dimensional time is observed.

Finally, and from the two approaches adopted here we can conclude that rotation affects not only the kinematic field of homogeneous turbulence but also the scalar field. After this work, we think that study of rotation effects remains also a difficult subject. Difficulties remaining in predicting kinematic and essentially scalar field We think that some inconsistency may exist in the formulation of modeled transport equations of dissipations rates of turbulent kinetics energy and scalar variance. To improve its prediction, we think also that a correction of coefficients of model 1 (LRR), to take into account correctly of rotation effects, can make another extension to this work.

A possible extension of the recent work is to homogeneous sheared turbulence submitted to both effects of rotation and stable stratification A second 
order modeling of such turbulence seems to be an important direction of investigation.

\section{REFERENCES}

Bouzaiane, M., H. Ben Abdallah and T. Lili (2004). A second order modeling of a stably stratified sheared turbulence submitted to a non vertical shear. Journal of turbulence 5.

Bouzaiane, M., H. Ben Abdallah and T. Lili (2003). A stably on the asymptotic behaviors of dimensionneless parameters in a stably homogeneous sheared turbulence. Journal of turbulence.

Brethouwer, G. (2005). The effect of rotation on rapidly sheared homogeneous turbulence and passive scalar transport. J Fluid Mechanic 542, 305-342.

Cadiou, A. (1996). A Contribution to the Study of Second Order Turbulence Closure Models. Ph.D. thesis, Central school of Nantes France.

Chebbi, B., M. Bouzaiane and T. Lili (2009). Prediction of equilibrium states of kinematic and thermal fields in homogeneous turbulence submitted to the rotation. International Symposium on Convective Heat and Mass Transfer in Sustainable Energy 200, 376- 379.

Chebbi, B., M. Bouzaiane and T. Lili (2007). Second order closur modeling of homogeneous sheared turbulence in a rotating frame, 18th French congress of Mechanics 2007-0711, Grenoble France.

Frank ,G. Jacobitz., Lukas Liechtenstein, Kai Shneider and Marie Farge (2008). On the structure and dynamics of sheared and rotating turbulence: Direct numerical simulation and wavelet-based coherent vortex extraction. Physics of Fluids 20, 045103-1.

Holt, S.E., J.R. Koseff, J.H. Ferziger (1992). Anumerical study of the evolution and structure of homogeneous stably stratified sheared turbulence. Journal Fluid Mechanic 237, 499-539.

Khaleghi, K., M.Pasandideh. Fard, M. Malek. Jafarian and Y.M Chung (2010).Assessment of Common Turbulence Models under Conditions of Temporal Acceleration in a Pipe. Journal of Applied Fluid Mechanics 3(1), 25-33.

Hanjalic, K. (1994). Advanced turbulence closure models: a view of current status and future prospects. Int. J. Heat and Fluid Flow 15(3).

Launder, B.E., G. Reece and W. Rodi (1975). Progress in the development of a Reynolds stress closure. Journal Fluid Mechanic 68, 537-576.

Poroseva, S. V., S.C. Kassinos, C.A. Langer, W.C. Reynolds (2002). Structure-based Turbulence model: application to a rotating pipe flow. Phys. Fluids 14, 1523-1532.

Ristorcelli, J.R., J.L. Lumley and R. Abid (1998). Rapid-pressure correlation representation consistent with the Taylor-Proudman theorem materially-frame-indifferent in the 2D limit. Institute for computer applications in science and engineering (ICASE), VA23681, NASA Langley research center, Hampton.

Schiestel, R. and L. Elena (1997). Modeling of anisotropic turbulence in rapid rotation. Aerospace Science and Thechnology 7(7), 4416451.

Shih, T., T.H . Constitutives (1996). Relations and Realisability of Single-Point Turbulence Closures, in TurbulenceTransition and Modeling. edited by Mr.Hallback D.S. Hennigson and A.V. Johansson and P.H. Alfredsson, Dordrech.

Shih, T., J. Chen and L.L. Lumley (1985a). Second order modeling of boundary-free turbulent shear flows with a new model form of pressure correlation. Rept. FDA-85-3, Sibley School of Mech. and Aerospace Eng., Cornell University.

Shih, T., J. Chen and L. Lumley (1985b). Modeling of pressure correlation terms in Reynolds-stress and scalar flux equations. Rept.FDA-85-3, Sibley School of Mech. And Aerospace Eng., Cornell University.

Sodja, J., R. Podgornik (2007). Turbulence models in $C F D$. University of Ljubljana Faculty for mathematics and physics.

Speziale, C.G. and N.M.G. Mhuris (1988). Scaling laws for homogeneous turbulent shear flows in a rotating frame. NASA Report 23665, NASA Langley research center, Hampton, Virginia.

Speziale, C.G. and T.B. Gatski (1997). Analysis and modeling of anisotropies in the dissipation rate of turbulence. Journal Fluid Mechanic 344, 15-180.

Speziale, C.G., S. Sarkar and T.B. Gatski (1990). Modeling the pressure strain correlation of turbulence an invariant dynamical systems approach. NASA Report 23665-5225, NASA Langley research center, Hampton, Virginia.

Speziale, C.G. and N.M.G. Mhiris (1989). On the prediction of equilibrium states in homogeneous Turbulence. Journal Fluid Mechanic 209, 591615.

Tavoularis, S. and S. Corrsin (1981). Experiments in nearly homogeneous turbulent shear flow with a uniform mean temperature gradient, Part1. Journal fluid mechanic 104, 311-347.

Wang, Y., K. Nagata and S. Komori (2000). Strongly stably stratified grid turbulence using second moment closure, A IAA J. 8, 31-38. 
B. Chebbi and M. Bouzaiane / JAFM, Vol. 5, No. 2, pp. 55-65, 2012.

\section{Appendix A. Derivation of Coefficients Associated to Analytical Solutions}

We give in this appendix expressions of coefficients associated to analytical solutions where effects of both viscosity and pressure are neglected.

$A_{11}=-\frac{(1-2 R) y_{2}(0)+2 R y_{1}(0)}{4 R}$

$C_{11}=y_{1}(0)+\frac{y_{4}(0)}{4 R}-A_{11}=y_{1}(0)-D_{11}$

$D_{11}=-A_{11}-\frac{y_{4}(0)}{4 R}$

$y_{1}(0)=\overline{u_{1}^{2}}(0) ; y_{2}(0)=\overline{u_{2}^{2}}(0) ; y_{3}(0)=\overline{u_{3}^{2}}(0)$;

$y_{4}(0)=\overline{u_{1} u_{2}}(0) ; y_{5}(0)=k(0)$

$C_{12}=\frac{(1-2 R) y_{2}(0)+2 R y_{1}(0)}{4 R_{i}}$

$D_{12}=y_{4}(0)-C_{12}$

$A_{22}=-4 R C_{12}$

$D_{22}=\frac{-y_{4}(0)+C_{12}}{2(1-2 R)}$

$C_{22}=y_{2}(0)+\frac{y_{4}(0)-C_{12}}{2(1-2 R)}=y_{2}(0)+\frac{D_{22}}{2(1-2 R)}$

$C_{33}=y_{3}(0)$

$C_{k}=y_{5}(0)+y_{4}(0)+C_{12}$

$D_{k}=y_{4}(0)+C_{12}$

$C_{1 \theta}=y_{6}(0)+\frac{\alpha D_{12}+(2 R-1) D_{2 \theta}}{4 R_{i}}-\frac{(2 R-1) E_{2 \theta}}{R_{i}}$

$A_{1 \theta}=(2 R-1) C_{2 \theta}-\alpha C_{12}$

$B_{1 \theta}=(2 R-1) A_{2 \theta}$

$D_{1 \theta}=\frac{(2 R-1) D_{2 \theta}-\alpha D_{12}}{4 R_{i}}$

$E_{1 \theta}=\frac{(2 R-1) E_{2 \theta}}{R_{i}}$

$C_{\theta \theta}=y_{8}(0)+\frac{2 \alpha D_{2 \theta}}{4 R_{i}}+\frac{E_{2 \theta}}{R_{i}}$

$A_{\theta \theta}=-2 \alpha C_{2 \theta}$

$B_{\theta \theta}=-2 \alpha A_{2 \theta}$

$D_{\theta \theta}=-\frac{2 \alpha D_{2 \theta}}{4 R_{i}}$

$E_{\theta \theta}=-\frac{E_{2 \theta}}{R_{i}}$

\section{Appendix B. Coefficients of the Shih and Lumley Models}

B.1 Coefficients of the Pressure- Strain Correlation

$\beta=2+\frac{8}{9} F\{1+62.4(-I I+2.3 I I I)\}$

$F=1+9 I I+27 I I I$

$I I=-\frac{1}{2} b_{i j} b_{i j}$

$I I I=\frac{1}{3} b_{i j} b_{j k} b_{k i}$

$\alpha_{5}=-\frac{1}{10}(1+8 \sqrt{F})$

B.2 Model of the Pressure-Temperature Correlation

$\phi^{\theta}=\frac{\beta}{2}+r_{c}-\left(\frac{(\beta-2) I I_{d} / 6}{I I_{d} / 3+b_{i j} d_{i j}^{2}-b_{i j} d_{i j}}\right)$

In this expression $\beta$ and $b_{i j}$ are already given and $I I_{d}$ and $d_{i j}$ will be as

$d_{i j}=\frac{\overline{\theta^{2}} \overline{u_{i} u_{j}}-\overline{\theta u_{i}} \overline{\theta u_{j}}}{\overline{\theta^{2}} \overline{q^{2}}-\overline{\theta u_{p}} \overline{\theta u_{p}}}$ and $I I_{d}=\frac{d_{i i}^{2}-d_{i j}^{2}}{2}$ where

$d_{i i}^{2}=d_{i j} d_{i j}$

$r_{c}$ is the ratio of kinematic rate to scalar rate, its

expression is $r_{c}=\frac{\overline{q^{2}} \overline{\varepsilon_{\theta}}}{\bar{\varepsilon}} \overline{\overline{\theta^{2}}}$.

$I_{i j k}$ is linear in $\overline{\theta u}_{i}$ and its form is

$I_{i j k}=0.4 \delta_{i j} \overline{\theta u}_{k}-0.1\left(\delta_{i k} \overline{\theta u}_{j}+\delta_{j k} \overline{\theta u}_{i}\right)+0.1 b_{i j} \overline{\theta u}_{k}$

$-0.3\left(b_{i k} \overline{\theta u}_{j}+b_{j k} \overline{\theta u}_{i}\right)+0.2 \delta_{i j} b_{k p} \overline{\theta u}_{p}$ 